\title{
MUSCULOSKELETAL PAIN IN BRAZILIAN SUGAR CANE
} CUTTERS

Maria Helena Marziale, ${ }^{1}$ Vanda Felli, ${ }^{2}$ Juliana Mazaro, ${ }^{1}$ Leila Sarquis, ${ }^{3}$ David Coggon ${ }^{1}$ University of Sao Paulo, Ribeirão Preto, Brazil; 'University of Sao Paulo, Sao Paulo, Brazil; ${ }^{3}$ Federal University of Parana, Curitiba, Brazil; ${ }^{4}$ University of Southampton, Southampton, UK

10.1136/oemed-2011-100382.221

Objectives The worker involved in the sugar cane cut is exposed to both physical and mental health risks, due to several body movements that demand intense physical effort, along with inappropriate posture and exposure to different climate conditions. These aspects have caused working accidents and sickness such as musculoskeletal problems. This study aimed to assess the prevalence of musculoskeletal symptoms in Brazilian sugar cane cutters, as part of the multi-national CUPID study.

Methods A cross-sectional survey was carried out in a target population of 300 sugar cane cutters at an alcohol and sugar factory in São Paulo, Brazil, during the 2009 harvest. The data were collected at interview, using the Portuguese version of the standardised CUPID questionnaire.

Results Interviews were completed with 182 (60.6\%) subjects, most of whom (94.5\%) were men. Their average age was 35 years, and $63,6 \%$ were younger than 41 years. $29.8 \%$ of the participating workers reported low back pain in the past year, $4.4 \%$ neck pain, $4.4 \%$ shoulder pain, $7.9 \%$ elbow pain, $2.3 \%$ wrist/hand pain, and $2.3 \%$ knee pain.

Conclusions Work as a sugar cane cutter is physically demanding, and their relatively low frequency of reported pain is therefore remarkable. Their employment was more precarious that that of many other occupational groups, and this may have led them to under-report musculoskeletal pain. 